SCIENCE CHINA

Physics, Mechanics \& Astronomy

\title{
The stress-velocity relationship of twinning partial dislocations and the phonon-based physical interpretation
}

\author{
YuJie Wei ${ }^{1,2 *}$, and ShenYou Peng ${ }^{1,2}$ \\ ${ }^{1}$ State Key Laboratory of Nonlinear Mechanics (LNM), Institute of Mechanics, Chinese Academy of Sciences, Beijing 100190, China; \\ ${ }^{2}$ School of Engineering Sciences, University of Chinese Academy of Sciences, Beijing 100049, China
}

Received May 9, 2017; accepted July 4, 2017; published online September 8, 2017

\begin{abstract}
The dependence of dislocation mobility on stress is the fundamental ingredient for the deformation in crystalline materials. Strength and ductility, the two most important properties characterizing mechanical behavior of crystalline metals, are in general governed by dislocation motion. Recording the position of a moving dislocation in a short time window is still challenging, and direct observations which enable us to deduce the speed-stress relationship of dislocations are still missing. Using large-scale molecular dynamics simulations, we obtain the motion of an obstacle-free twinning partial dislocation in face centred cubic crystals with spatial resolution at the angstrom scale and picosecond temporal information. The dislocation exhibits two limiting speeds: the first is subsonic and occurs when the resolved shear stress is on the order of hundreds of megapascal. While the stress is raised to gigapascal level, an abrupt jump of dislocation velocity occurs, from subsonic to supersonic regime. The two speed limits are governed respectively by the local transverse and longitudinal phonons associated with the stressed dislocation, as the two types of phonons facilitate dislocation gliding at different stress levels.
\end{abstract}

dislocation mobility, transverse and longitudinal phonons, subsonic and supersonic velocity, stress-velocity relationship, molecular dynamics

PACS number(s): 63.20.-e; 61.72.Lk; 71.15.Pd

Citation: Y. J. Wei, and S. Y. Peng, The stress-velocity relationship of twinning partial dislocations and the phonon-based physical interpretation, Sci. China-Phys. Mech. Astron. 60, 114611 (2017), doi: 10.1007/s11433-017-9076-8

\section{Introduction}

Dislocations are line defects which are the most commonly seen carriers for plastic deformation in crystalline metals. The mobility of dislocations directly influences the strength and ductility of crystalline materials [1-5]; Its significance is evidently seen in the relation $\dot{\varepsilon}=\rho_{\mathrm{d}} b v$ proposed by Orowan [3], from which the macroscopic plastic deformation rate $\dot{\varepsilon}$ in crystals is directly related to dislocation density $\rho_{\mathrm{d}}$, dislocation velocity $v$, and the Burgers vector $b$ of a dislocation. The

*Corresponding author (email: yujie_wei@Inm.imech.ac.cn) exact dependence of dislocation velocity $v$ on resolved shear stress $\tau$, while being the fundamental ingredient for deformation map in most crystalline materials [4,5] and associated with critical phenomena like crack propagation $[6,7]$ and brittle-ductile transition [8], is however not fully understood. While recording static dislocations even with sub-angstrom spatial resolution is practical [9], tracking a moving dislocation with fine spatial and temporal resolution is challenging [10]. At low stress level $\tau$, a general empirical relation for dislocation velocity $v, v=v_{0}\left(\tau / \tau_{0}\right)^{m} \exp \left(-Q / k_{\mathrm{B}} T\right)$, has been broadly used $[5,11,12]$. Here $v_{0}$ is related to the velocity of a dislocation at the reference resolved shear stress $\tau_{0}, m$ is known as a dislocation velocity-stress exponent and $Q$ is the 
activation energy for dislocation motion, $k_{\mathrm{B}}$ is the Boltzmann constant and $T$ is the absolute temperature. Such a relationship resembles or possibly originates from the typical powerlaw strain rate sensitivity equation of $\dot{\varepsilon}=\dot{\varepsilon}_{0}\left(\tau / \tau_{0}\right)^{1 / m}$, for $\dot{\varepsilon}_{0}$ a reference strain rate at $\tau_{0}$. Regardless its common appearance in textbooks, there lacks critically examination on the validity of the empirical stress-velocity relationship of an individual dislocation from either experiments or simulations.

In addition to the concern on the validity of the empirical stress-velocity relationship of dislocations, another issue emerges as the resolved shear stress continues to increase: where is the limit of the dislocation speed $v$ ? The empirical equation does not predict the behavior of dislocations when the stress is sufficiently high but we know for sure that $v$ is bound at some level. By arguing that the speeds of dislocations are limited by the inertia of the elastic fields, Frank [13] and Eshelby [14] concluded that the speeds of the dislocations are bound by the shear wave speed. Based on simplified lattice interactions, Earmme and Weiner [15] showed that there is a breakdown phenomenon in high speed dislocations. Later on fully three-dimensional atomistic simulations, however, suggested that dislocation velocity may exceed the shear wave speed in certain conditions [7,16-18]. Theoretical analysis by Rosakis [19] also suggested the possibility of dislocation speed exceeding the shear wave speed, with support from recent experiments [20-22]. It is noted, however, there are remaining doubts on the existence of supersonic dislocations [23]. All these confusions reflect the compelling need of knowing the physical origin of dislocation speed limit. Conclusive evidence has been lacking because realizing well-defined experimental measurements to capture spatial and temporal information of moving dislocations is still not attainable. Technically, existing reports on the velocity of dislocations were abstracted from post-mortem dislocation patterns $[21,24,25]$. The velocity was averaged within the observer's time window. Hence it was not the transient velocity of a stressed dislocation but relied heavily on the time span between recorded dislocation patterns. The most recent work by Nosenko et al. [20,22] can be regarded as in-situ. The authors abstracted the distance-time data from direct video observations during dislocation motion in plasma crystals, where their shear wave speeds are typically on the order of tens of millimetres per second, orders of magnitude lower than those of dislocations in metals.

\section{Simulation methods}

Powered by rapid growth of computational capability, molecular dynamics (MD) simulations supply an alternative tool to explore novel deformation mechanisms under extreme conditions [26-31]. We adopt this tool to explore the stress dependent dislocation mobility. For generality, we consider a MD sample with twinning partial dislocations in face-centered cubic (F.C.C.) metals residing in twin planes, as shown in Figure 1(a). In F.C.C. crystals with pre-existing twin planes, plasticity by twin growth or detwinning is typically achieved via passing Shockley partial dislocations with a $a / 6(112)$ burgers vector over every $\{111\}$ plane above the twinning plane [32].

The dimensions of the periodic simulation box for a twinning partial dislocation quadruple are $L_{x}=1825 \mathrm{~nm}$, $L_{y}=456 \mathrm{~nm}$, and $L_{z}=0.512 \mathrm{~nm}$. Sufficiently large simulation box is desired in order to eliminate possible size effect caused by interactions among the periodic arrangement of dislocations or image stress due to surface effect in finite samples in vacuum, as illustrated by Bhate et al. [33]. The large space between dislocations ensures them to reach their speed limit before collision and annihilation, and also helps to minimize their mutual interaction. In addition, it ensures an accurate quantification of the resolved shear stress applied to dislocations even after the dislocations travel for a long distance. The $x$-, $y$ - and $z$-directions are respectively parallel to [11 $\overline{2}],[111],[\overline{1} 10]$ crystallographic directions in the single crystalline sample with twin planes. Typical samples contain about 35000000 atoms. To realize periodic boundary conditions and to minimize long-range interaction between dislocations and their images, we design a dislocation quadruple in our MD samples, as illustrated in Figure 1(b). Dislocations of opposite sign along the $x$ and $y$-axis ensure periodic boundary conditions along both directions. The pairs along $x$-axis may glide to each other. When they encounter, dislocation annihilation occurs. The positions of the obstacle-free dislocations are recorded as we continuously apply shear strain to the sample. The velocity-stress relationship is deduced from the position-time history of the dislocations. Since there are four dislocations, there should be four values of velocity of dislocation. With the geometrical symmetry, the speeds of the four dislocations should be the same. We take the average value of the four as the final velocity of the dislocations.

MD simulations are performed in large-scale atomic/molecular massively parallel simulator (LAMMPS) [34]. Periodic boundary conditions are applied in the $x$-, $y$ and $z$-directions. Interactions among atoms are captured by the embedded atom method (EAM) potentials [35]. We use the potential developed by Mishin et al. [36] for copper; potential for aluminum was from Zhou et al. [37]; and potentials for gold, silver, nickel, platinum, and palladium were developed by Foiles et al. [38]. Before applying strain-rate controlled shearing to the samples, we relax the initial structures through controlling both pressure and temperature (NPT ensemble). After relaxation, we apply shear deformation to the samples at a constant strain rate. We adopted a microcanonical ensemble so that the macroscopic variables $(N, V, E)$ of the microcanonical ensemble are 

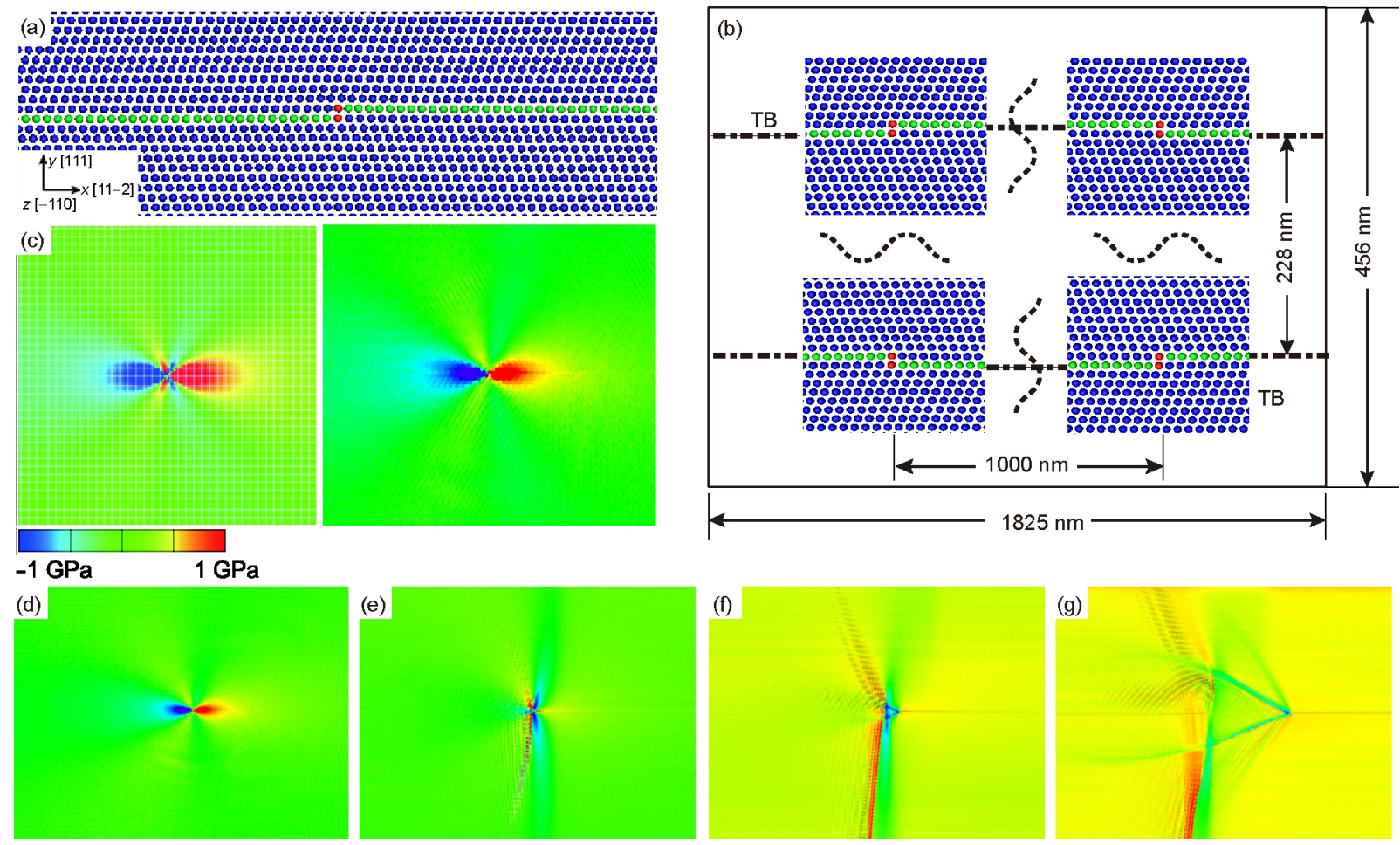

Figure 1 (Color online) The simulation model for twinning partial dislocations in face-centered cubic metals. (a) The atomistic structure of a twinning partial dislocation (red atoms) residing in a twin boundary (green atoms); (b) the designed sample with four dislocations (a dislocation quadruple) in the simulation box to ensure periodic boundary conditions; (c) the stress field $\sigma_{x y}$ induced by a static twinning partial dislocation in $\mathrm{Cu}$ from theoretical prediction (left) and MD simulation (right); (d) to (g) $\sigma_{x y}$ introduced by the moving twinning dislocation at different velocity (shearing rate: $2 \times 10^{8} / \mathrm{s}$ ); (d) before reaching its first speed limit $v=800 \mathrm{~m} / \mathrm{s}$; (e) moving at the first speed limit $v=1831 \mathrm{~m} / \mathrm{s}$; (f) accelerating to the second limit $v=3000 \mathrm{~m} / \mathrm{s}$; (g) moving at the second limit $v=4463 \mathrm{~m} / \mathrm{s}$.

constants, where $N$ is the total number of particles in the system, $V$ is the system's volume, and $E$ is the total energy in the system. The time step is set to be 0.5 femtosecond. As a result of the chosen ensemble, there is a slight temperature rise in the sample subjected to tension.

\section{Results and discussions}

The dislocations (Figure 1(a)) we simulated are of edge type. The theoretical stress contour $\sigma_{x y}$ induced by an edge dislocation sitting in $(0,0)$ with its Burgers vector parallel to the $x$-axis is shown in Figure 1(c) (left), in contrast we show the result from MD simulation as well. Figure 1(e)-(h) shows the corresponding shear stress $\sigma_{x y}$ resulted from the dislocation moving at different velocity. Here we apply constant shear strain rate $2 \times 10^{8} / \mathrm{s}$ to the sample shown in Figure $1(\mathrm{~b})$. The shear stress contour of the twinning partial dislocation moving at $v=800 \mathrm{~m} / \mathrm{s}$ (Figure $1(\mathrm{e})$ ) is very similar to that at rest (Figure 1(c)). When it reaches the first speed limit of $v=1831 \mathrm{~m} / \mathrm{s}$, the stress field seems to be compressed within a narrow region (Figure 1(f)). Further increasing in shear stress will drive the dislocation moving faster than the shear wave speed. From Figure 1(g), we see the embark of wave front at the speed of $v=3000 \mathrm{~m} / \mathrm{s}$. A steady shear wave front is seen when the dislocation reaches its second limit of $v=4463 \mathrm{~m} / \mathrm{s}$ (Figure 1(h)). The dynamic shear stress field at different velocity can be viewed from the movie (see the supporting information (SI-1)).

To obtain an accurate relationship between the resolved shear stress and dislocation velocity, we record dislocation motion within the elastic regime, where the applied shear stress increases linearly with shear strain, as clearly seen in Figure 2(a). Figure 2(b)-(d) show the travelled distance-stress and the corresponding velocity-stress curves of the twinning partial dislocation, at the strain rates of $2 \times 10^{7} / \mathrm{s}, 2 \times 10^{8} / \mathrm{s}$, $2 \times 10^{9} / \mathrm{s}$, respectively. As the applied shear stress increases, we see two slopes (for both strain rates at $2 \times 10^{8} / \mathrm{s}$ and $2 \times 10^{9} / \mathrm{s}$ ) where the travel distances increase linearly with stress. The dislocation moves at a constant velocity within a certain stress regime. From the deduced velocity-stress curves, we see that the speed of the twinning partial increases rapidly at low stress level. The stress-velocity relation shown in Figure 2(b)-(d) indicates that the minimum value of $\tau_{f}$ to overcome lattice friction and to drive dislocation motion in a close-packed plane is on the order of $10 \mathrm{MPa}$. Theoretical estimate with $\tau_{f}=\frac{2 G}{(1-v)} \exp \left[-\frac{2 \pi b}{h(1-v)}\right][39,40]$ gives a threshold resistance on the order of $\tau_{f} \approx 160 \mathrm{MPa}$ in $\mathrm{Cu}$ with $G=46 \mathrm{GPa}, v=0.34, b=0.15 \mathrm{~nm}$, and $h=0.21 \mathrm{~nm}$. As 

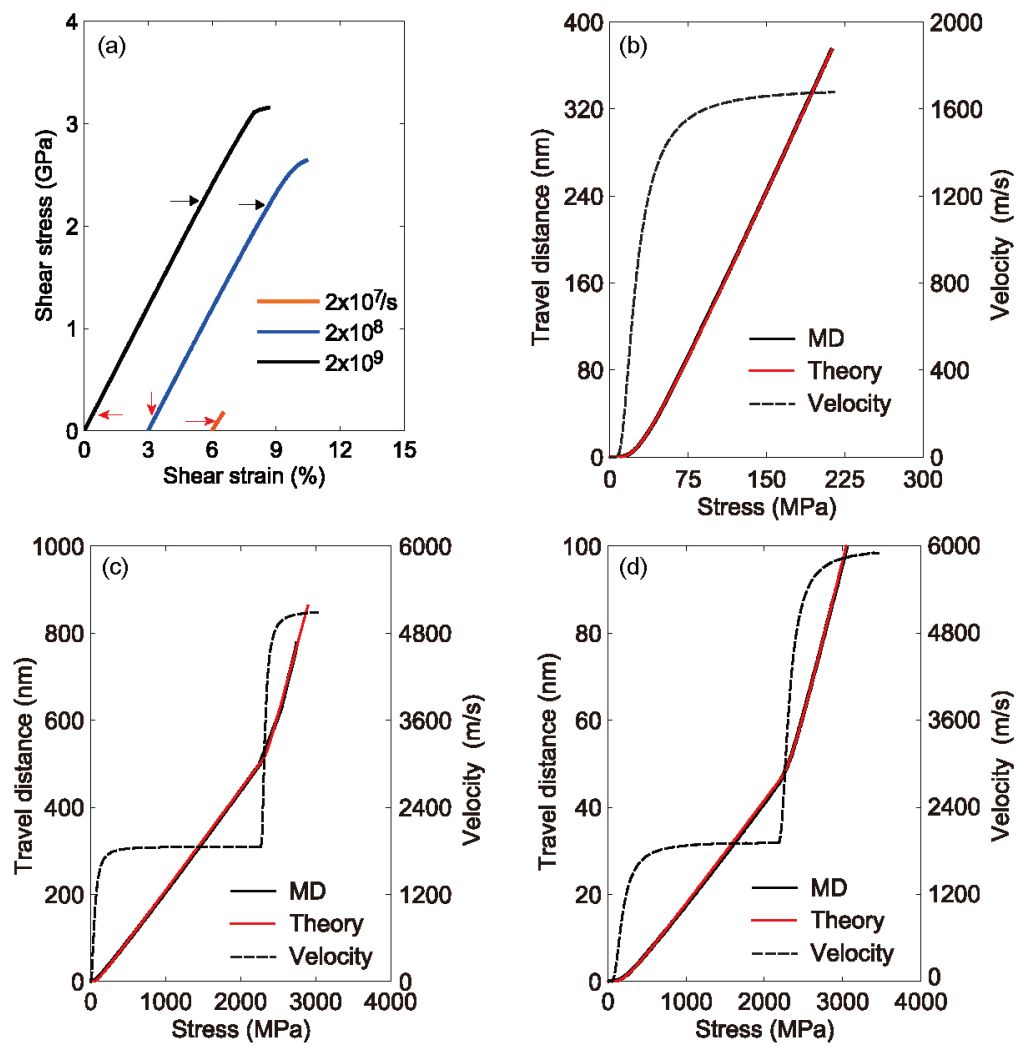

Figure 2 (Color online) The velocity of twinning dislocations in $\mathrm{Cu}$ at different strain rates. (a) The stress-strain curves from molecular dynamics simulations. Red arrows point to the critical stress levels when the first velocity limit approaches, and black arrows refer to the transitional point for the second velocity limit; (b)-(d) the travel distance and the velocity of the twinning dislocation at different strain rates: (b) $2 \times 10^{7} / \mathrm{s}$, (c) $2 \times 10^{8} / \mathrm{s}$, and (d) $2 \times 10^{9} / \mathrm{s}$.

Nabarro [40] pointed out, the prediction is so sensitive to the details of the model that its value is only heuristic. Our MD results suggest that the stress required to move Shockley twinning partial dislocations lying in twin planes may be rather small. We also show the theoretically predicted displacement-stress curve at different strain rates, which will be detailed late.

We show the stress field $\sigma_{x x}$ and the horizontal velocity field induced by the twinning partial dislocation at snapshots within different speed region. The applied shear strain rate is $2 \times 10^{8} / \mathrm{s}$. Figure 3(a)-(d) shows the snapshots of $\sigma_{x x}$ induced by the twinning partial moving at $v=800,1831,3000,4463 \mathrm{~m} / \mathrm{s}$, respectively. The shock wave induced by the supersonic dislocation is clearly seen as the dislocation speeds up from the first velocity limit (Figure 3(a) and (b)) to the second speed limit (Figure 3(c)). When the dislocation moves at the second limiting speed, it introduces a steady shock wave front moving with the dislocation (Figure 3(d)). The movie in SI-2 shows the whole dynamic process. In Figure 3(e)-(h), we also show the horizontal velocity contours at the dislocation speeds corresponding to Figure 3(a) and (d), respectively. The same trend is observed: the dislocation moving at the second speed limit produces a shock wave in terms of the horizontal speed of atoms. A shear-wave Mach cone, the distinct signature resulting from the motion of dislocation at a velocity faster than the shear wave speed, is clearly seen in Figure 3(d) and (h). The corresponding movie to show the horizontal velocity contour is given in SI-3. We also show in SI-4 the pressure contours.

It is clearly demonstrated in our MD simulations that a twinning partial dislocation can move faster than the material's shear wave speed at high stress. However, the behind physical origin governing the motion of dislocations remains controversial. The previous empirical relationship for dislocation velocity $v$ and stress $\tau$ in the form of $v \propto \tau^{m}$ was essentially proposed to fit the experimental results. Johnston and Gilman [11] suggested that $m$ ranges from 15 to 25 based on dislocation-velocity data in lithium fluoride crystals. Greenman et al. [24] showed experimentally that for $\mathrm{Cu} m=0.7$. For Al, Gorman et al. [41] reported a linear dependence of $v$ on $\tau$ at a low speed region. The measurement by Yasutake et al. [25] for twinning partial dislocations in silicon suggests a distinct dependence of dislocation velocity on stress, which is better captured with $m=2.73$. The significant scattering of $m$ reflects the weakness of the mathematical formula which lacks correlation with physical mechanisms. In addition, accompanied by the rapid increase of velocity in response to stress increase, the velocity of dislocations saturates within a wide stress regime. Although different models were proposed to capture the saturated speed as well, the exact formula is typically a priori of the known shear speeding limit [42]. All 

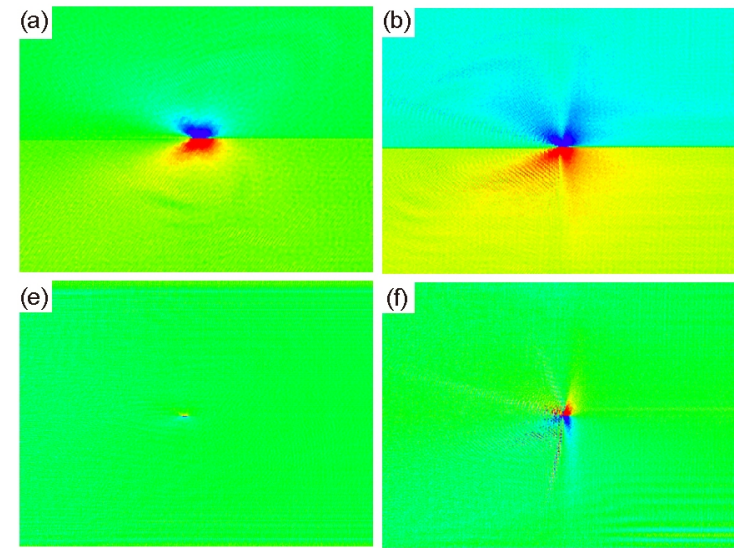

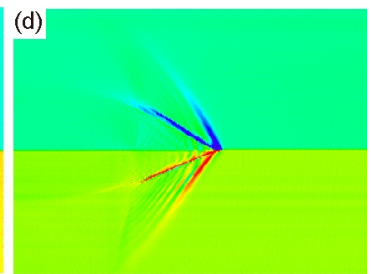

(h)

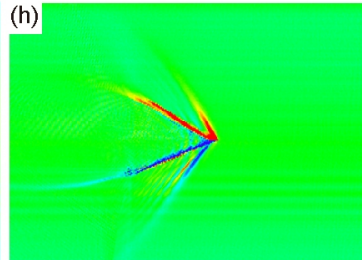

Figure 3 (Color online) The stress field $\sigma_{x x}$ and the horizontal velocity field induced by the twinning partial dislocation at snapshots with different velocity (shearing rate: $2 \times 10^{8} / \mathrm{s}$ ). (a)-(d) Stress fields $\sigma_{x x}$, (a) before the first speed limit $v=800 \mathrm{~m} / \mathrm{s}$, (b) moving at the first speed limit $v=1831 \mathrm{~m} / \mathrm{s}$, (c) before the second speed limit $v=3000 \mathrm{~m} / \mathrm{s}$, (d) moving at the second speed limit $v=4463 \mathrm{~m} / \mathrm{s}$, (e)-(h) show in turn the horizontal velocity contours corresponding to (a)-(d).

these observations call for the development of physically sound models which could capture the velocity-stress relationship seen in Figure 2.

\section{$4 \quad$ Theoretical modelling}

To find the physical origin of the dislocation velocity-stress dependence, we examine the energy profile as a dislocation glides along a lattice. Figure 4(a) presents the lattice energy profile as proposed by Peierls [39]. With thermal undulation or stress assistance, both lattice and the dislocation will vibrate [43]. When the normal mode perfect lattice vibration meets dislocation vibration, it may result in drag on dislocation by phonon scattering, or combine with the applied stress to promote dislocation motion [44]. The lattice vibration for atoms in the dislocation core can be categorized into longitudinal mode and transverse mode. For the former, the displacement of atoms from their equilibrium positions coincides with the wave propagation direction (Figure 4(c)); in the transverse mode, atoms move perpendicularly to the dislocation sliding plane (Figure 4(b)). Atoms shifted from their equilibrium positions will modify their energy status. As illustrated in Figure 4(d) and (e), the displacement of the atom in the dislocation core resulted from transverse and longitudinal vibrations lowers the energy barrier for dislocation move, and the phonon states related to lattice vibrations change accordingly.

Now we consider a phonon mode of frequency $\omega$. Following the same way for phonon modes triggered by thermal energy, we consider phonon modes of stressed lattice whose distribution follows the Planck distribution [45]: $\langle n\rangle=\frac{1}{\exp \left(\frac{\hbar \omega}{\tau \Omega_{a}}\right)-1}$. Here $\langle n\rangle$ is the average phonon number with frequency $\omega$; the thermal energy term in the partition function is replaced by the stress energy term at $0 \mathrm{~K}$, for $\hbar$ the Planck constant, $\tau$ the average resolved shear stress applied to the dislocation, and $\Omega_{a}$ the volume of the dislocation core. We consider stress modified phonon distribution by making the following analogy: the stress fluctuates all the time as temperature does but its ensemble approaches to a constant value. The change in phonon distribution by stress is not governed by the release of strain energy but by the fluctuation of stress. Hence we choose the work-conjugate term $\Omega_{a}$ and let it a physical equivalence to the thermal energy. In Appendix A, we give an explanation on the physical equivalence between stress and temperature on how they may alter phonon distribution.

For simplicity, we use the Einstein model of the density of states [46]: all $N$ oscillators within the region of interest have the same frequency $\omega$. The phononic density of state function is then given as $D(\omega)=N \delta\left(\omega-\omega_{0}\right)$. The collective energy of the $N$ oscillators is $e=\frac{N \hbar \omega}{\exp \left(\frac{\hbar \omega}{\tau \Omega_{a}}\right)-1}$. It is noted that we would not expect the Planck distribution of phonon modes or the Einstein model of the density of states fully capture the real phonon characteristic in our MD systems. Even simple cubic lattices with nearest neighbor interaction exhibit complicated phonon distribution in defective solids [47-50], and the models employed by the most represent a first-order approximation of phonon features. Nevertheless, the models are conceptually convenient for one to understand the physical origin of the proposed velocity-stress relationship of dislocations. Accompanying with the energy-barrier change as the dislocation glides through a lattice site (Figure 4), phonons annihilate and regenerate. Such processes result in energy dissipation. The dissipated energy is comparable to the collective energy of the $N$ oscillators, as the fraction of plastic work converted to heat is about $80 \%$ or even higher during dislocation dominated plasticity $[51,52]$. Hence the energy- 

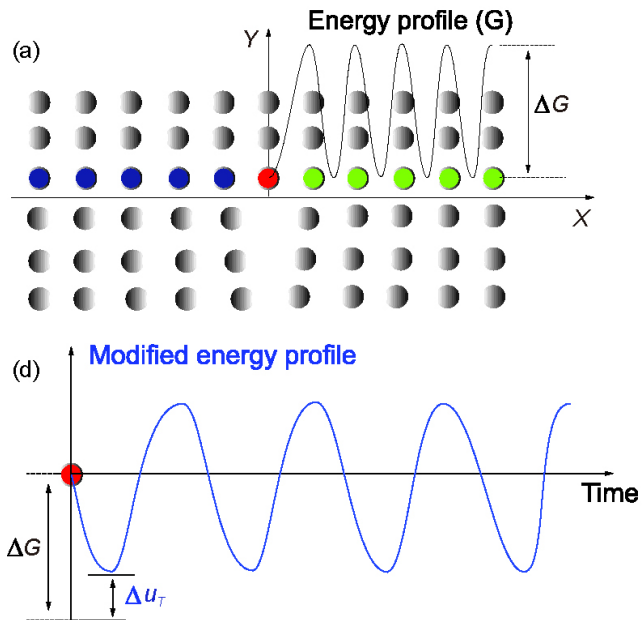

(b) Transverse phonon, $Y(t)$

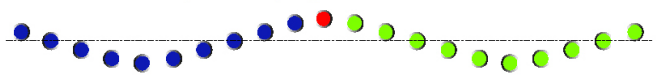

(c) Longitudinal phonon, $X(t)$

$\bullet \bullet \bullet \bullet \bullet \bullet \bullet \bullet 0000000000$

(e) Modified energy profile

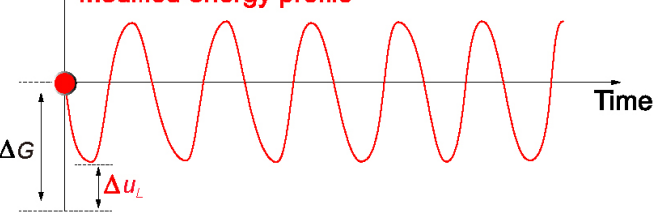

Figure 4 (Color online) Illustration to show the energy profile modified by transverse and longitudinal phonons modes as a dislocation glides. (a) Location of the dislocation and the periodic energy profile along the slip line. The original energy barrier $\Delta G$ may be modified by transverse and longitudinal phonons; (b) and (c) the transverse and longitudinal phonons introduce $y$ - and $x$-axis displacements, respectively, with the former being perpendicular to the Burgers vector and the latter being parallel to the Burgers vector; (d) and (e) the amount of lowered energy barrier by two types of phonons, where we have $\Delta u_{T}$ by the transverse phonon and $\Delta u_{L}$ by longitudinal phonon.

dissipation rate by dislocation gliding approximates to the variation rate of energy from the $N$ oscillators, which leads to the equality $\tau_{T} b L v=\mathrm{d} e_{T} / \mathrm{d} t$, where $b$ is the Burgers vector of the dislocation, $L$ the dislocation length, and $\tau_{T}$ the average frictional resistance to dislocation gliding with assistant from transverse phonons. In that circumstance, $e_{T}=\frac{N \hbar \omega_{T}}{\exp \left(\frac{\hbar \omega_{T}}{\tau \Omega_{a}}\right)-1}$, where $\omega_{T}$ is the frequency of transverse phonons. The velocity of the dislocation is then deduced as:

$v=\dot{\varepsilon} \frac{N \Omega_{a}}{b L} \frac{G}{\tau_{T}}\left(\frac{\hbar \omega_{T}}{\tau \Omega_{a}}\right)^{2} \frac{\exp \left(\frac{\hbar \omega_{T}}{\tau \Omega_{a}}\right)}{\left[\exp \left(\frac{\hbar \omega_{T}}{\tau \Omega_{a}}\right)-1\right]^{2}}$.

Since dislocation-velocity transition occurs within the linear elastic regime in the stress-strain curve seen in Figure 2(a), we have used $\tau=G \dot{\varepsilon} t$ while obtaining eq. (1), with $G$ being the shear modulus, $\dot{\varepsilon}$ the shearing rate and $t$ the time. The result given in eq. (1) is for the special case when the macroscopic stress change and microscopic stress fluctuation coincide and we have $\mathrm{d} \tau / \mathrm{d} t=G \dot{\varepsilon}$. For more general cases we would not see $\mathrm{d} \tau / \mathrm{d} t \propto \dot{\varepsilon}$, for example the activation of mechanisms which could dissipate large amount of work during deformation. In that case, the dependence of dislocation velocity has to be deduced from $\tau_{T} b L v=\mathrm{d} e_{T} / \mathrm{d} t$, which gives

$v=\frac{N \Omega_{a}}{b L \tau_{T}}\left(\frac{\hbar \omega_{T}}{\tau \Omega_{a}}\right)^{2} \frac{\exp \left(\frac{\hbar \omega_{T}}{\tau \Omega_{a}}\right)}{\left[\exp \left(\frac{\hbar \omega_{T}}{\tau \Omega_{a}}\right)-1\right]^{2}} \frac{\mathrm{d} \tau}{\mathrm{d} t}$.

At constant macroscopic stress, $\mathrm{d} \tau / \mathrm{d} t$ is related to the stress-fluctuation in the vicinity of the dislocation core and is non-zero. A one-step gliding of the dislocation would relax the stress within the activation volume. It takes a characteristic time $t_{\mathrm{c}}$ for the macroscopic constant stress to restore the stress in the activation volume. Therefore, we have $\frac{\mathrm{d} \tau}{\mathrm{d} t}=\tau / t_{\mathrm{c}}$. For $\tau \Omega_{a} \gg \hbar \omega_{T}$, eq. (2) is simplified to

$v=\frac{N \Omega_{a}}{b L \tau_{T}} \frac{\tau}{t_{\mathrm{c}}}$

The above equation predicts a linear dependence of velocity on stress when $\tau \Omega_{a} \gg \hbar \omega_{T}$. In contrast to the one-step loading (continuously increasing strain) in previous simulations, we evaluate eq. (3) by looking at the motion of dislocations subjected to a two-step loading: we first increase the strain at a constant strain rate; after the stress in the sample reaches a target value, we then keep it constant. In Figure 5(a) and (b), we show the distance-time and also the velocity-time curves of a dislocation at several stresses. The two figures correspond to two strain rates before reaching the target stress, $\dot{\varepsilon}=2 \times 10^{9} / \mathrm{s}$ and $2 \times 10^{7} / \mathrm{s}$, respectively. After a transient region, the velocity of the dislocation dwelling on a constant stress level is also a constant. It is consistent with eq. (3). However, when the stress level is so high and the dislocation moves at its limit speeds, the variation in dislocation velocity is very small even the stress difference is large, as shown in Figure 5(c). It is also interesting to see that the transient region at high strain rate is significant but it disappears at the strain rate of $\dot{\varepsilon}=2 \times 10^{7} / \mathrm{s}$, as seen in Figure 5(a) and (b). The discrepancy between velocities from the one-step and the two-step loading at different strain rates is clearly demonstrated in Figure 5(d). The final stable velocity during two-step loading overlaps with the velocity from one-step loading at a constant stress. Therefore, in order to examine 
the validity of eq. (3), a strain rate around $\dot{\varepsilon}=2 \times 10^{7} / \mathrm{s}$ or even lower is required.

From eq. (1), we see that the dislocation will reach its first speed limit as $\tau$ increases continuously. That speed could be obtained from extrapolating $\tau$ to a sufficiently large value $\tau_{\mathrm{c}}$ so that $\hbar \omega_{T} / \tau_{\mathrm{c}} \Omega_{a}$ is small. The first speed limit is given as $v_{T m}=\beta_{T} b \dot{\varepsilon} \frac{G \Omega_{a}}{\hbar \omega_{T}}$ with $\beta_{T}=\frac{N \hbar \omega_{T}}{b^{2} L \tau_{T}}$. Here we have made an assumption that at the stress level $\tau<\tau_{\mathrm{c}}$, transverse phonons are the primary assistance to dislocation gliding. Hence $\beta_{T}$ is a dimensionless coefficient pertinent with dislocation motion assisted by transverse phonons. Integrating eq. (1) with respect to time, we obtain the travelling distance $u_{T}$ of the dislocation as the shear stress increases linearly from 0 to $\tau$ :

$u_{T}=\frac{\beta_{T} b}{\exp \left(\frac{\hbar \omega_{T}}{\tau \Omega_{a}}\right)-1}$.

The velocity and the travel distance as a function of stress from MD simulations could be well captured by eqs. (1) and (4), as seen in Figure 2(b)-(d). Material parameters used to fit the curves at different strain rates are supplied in Table 1.
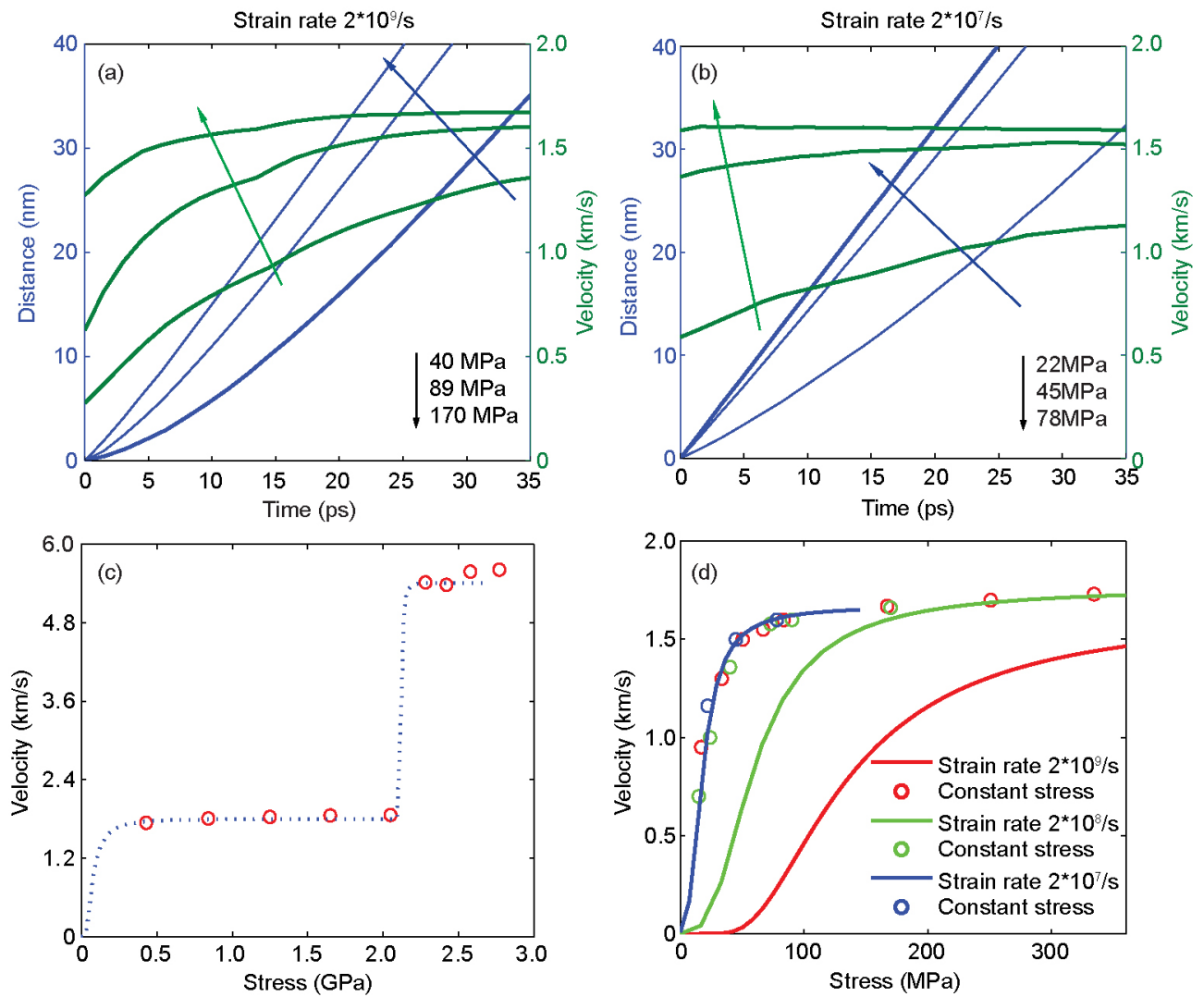

Figure 5 (Color online) Dislocation motion at a constant stress. (a) and (b) The displacement-time and corresponding velocity-time curves of a dislocation subjected to a constant stress at $\dot{\varepsilon}=2 \times 10^{9} / \mathrm{s}$ and $\dot{\varepsilon}=2 \times 10^{7} / \mathrm{s}$, respectively; (c) velocities in the saturated region at the strain rate of $\dot{\varepsilon}=2 \times 10^{8} / \mathrm{s}$. The dashed line comes from one-step loading and circles are dislocation velocities at constant stresses with two-step loading; (d) velocities in the accelerating region at three different strain rates, $2 \times 10^{9} / \mathrm{s}$ in red, $2 \times 10^{8} / \mathrm{s}$ in green, and $2 \times 10^{7} / \mathrm{s}$ in blue, respectively. Circles of the same color are the respective stable velocity under constant stresses.

Table 1 Material parameters for $\mathrm{Cu}$ to fit the dislocation travel distance and dislocation velocity as a function of stress. Here $v_{\mathrm{s}}=\sqrt{G / \rho}=2280 \mathrm{~m} / \mathrm{s}$ and $v_{1}=\sqrt{(B+4 G / 3) / \rho}=4627 \mathrm{~m} / \mathrm{s}$ are the theoretical velocities of the transverse sound (shear wave) and the longitudinal sound, respectively. The numbers are obtained by using the shear modulus $G=46 \mathrm{GPa}$, the Poisson's ratio $v=0.34$ and the density of $\rho=8.9 \times 10^{3} / \mathrm{m}^{3}$, and $\Omega_{a}=11.46 \AA^{3}$ for isotropic polycrystalline $\mathrm{Cu}$. It is noted that as the sample we used in MD simulation is a single crystal. The comparison between theoretical limits $v_{\mathrm{s}}$ and $v_{1}$ against their counterparts from MD simulations $v_{\mathrm{s}}^{\mathrm{md}}$ and $v_{1}^{\mathrm{md}}$ is for reference

\begin{tabular}{ccccccc}
\hline$\dot{\varepsilon}(1 / \mathrm{s})$ & $\omega_{T}$ or $\omega_{T}(1 / \mathrm{s})$ & $\beta_{T}$ & $\beta_{L}$ & $v_{\mathrm{s}}^{\mathrm{md}}(\mathrm{m} / \mathrm{s})$ & $v_{\mathrm{s}}^{\mathrm{md}} / v_{\mathrm{s}}$ & $v_{1}^{\mathrm{md}} / v_{1}$ \\
\hline $2 \times 10^{7}$ & $1.36 \times 10^{12}$ & 162 & - & 1696 & 0.75 & - \\
$2 \times 10^{8}$ & $3.5 \times 10^{12}$ & 45 & 56.5 & 1831 & 0.8 & 0.91 \\
$2 \times 10^{9}$ & $1.0 \times 10^{13}$ & 13.3 & 26.5 & 1894 & 0.83 & 5667 \\
\hline
\end{tabular}


Before the second speed transition, the first speed limit could sustain for a wide stress span when the stress increases from $\tau_{\mathrm{c}}$. Geometrically, the transverse phonon helps the atom at the dislocation core to circumvent its neighbours (moving perpendicular to the gliding plane, Figure 4(b)) and realize a successful jump, in contrast to the longitudinal phonon which helps the atom to push its neighbour aside and realizes an equivalent jump (Figure 4(c)). In the latter scenario, much high stress is required. At that high stress level, both the transverse mode and the longitudinal mode of dislocation motion are activated. This synergistic effect leads to an abrupt increase in the speed (Figure 2(c) and (d)). Note that even at low stress, transverse mode and longitudinal mode phonons may present concurrently. We neglect the longitudinal phonons below the first limiting velocity for the consideration that the majority of longitudinal phonons are triggered at a higher stress level. In the Einstein model, the transverse phonons and the longitudinal phonons are distributed at two separate points. In that sense, the two constant phonon frequencies may represent the collective effects of the exact distribution of phonons of each type. Such a treatment certainly deviates from the real case but the physical meaning remains. Now following the same analytical method and adding the contribution from longitudinal phonons assisted dislocation motion, with $e_{L}=\frac{M \hbar \omega_{L}}{\exp \left(\frac{\hbar \omega_{L}}{\tau \Omega_{a}}\right)-1}$, for $M$ the number of longitudinal phonons of frequency $\omega_{L}$, it is straightforward to deduce the stress dependent velocity when $\tau>\tau_{\mathrm{c}}$ :

$v=v_{T m}+\dot{\varepsilon} \frac{M \Omega_{a}}{b L} \frac{G}{\tau_{L}}\left(\frac{\hbar \omega_{L}}{\widehat{\tau} \Omega_{a}}\right)^{2} \frac{\exp \left(\frac{\hbar \omega_{L}}{\widehat{\tau} \Omega_{a}}\right)}{\left[\exp \left(\frac{\hbar \omega_{L}}{\widehat{\tau} \Omega_{a}}\right)-1\right]^{2}}$

with $\widehat{\tau}=\tau-\tau_{\mathrm{c}}$, where $\tau_{L}$ is the average frictional resistance to dislocation gliding with assistant from longitudinal phonons. The stress $\tau_{\mathrm{c}}$ is related to the critical condition when the majority of longitudinal phonon modes will be activated. It implies that the dislocation should be pushed at a high stress level to achieve the velocity jump. We expect that the role played by the applied force dominates over that by transverse phonons at the transition. Hence we should have $\tau_{\mathrm{c}} \Omega_{a}>\hbar \omega_{T}$. That is consistent with our simulation result that $\tau_{\mathrm{c}} \Omega_{a} / \hbar \omega_{T}$ is on the order of 2 to 5 . The second speeding limit, by extrapolating $\tau$ to $\infty$, is obtained as $v_{L m}=\frac{\dot{\varepsilon} G \Omega_{a} b}{\hbar}\left(\frac{\beta_{T}}{\omega_{T}}+\frac{\beta_{L}}{\omega_{L}}\right)$, with $\beta_{L}=\frac{M \hbar \omega_{L}}{b^{2} L \tau_{L}}$. It is seen that $v_{L m}$ depends on both the longitudinal and the transverse phonon properties of the material, while the first one $v_{T m}$ only depends on the shear properties. Both of them, however, are independent on the applied resolved shear stress. Integrating eq. (5) with time, we obtain the displacement-stress relationship as:

$$
u_{L}=u_{T 0}+\beta_{T} b \frac{\left(\tau-\tau_{\mathrm{c}}\right) \Omega_{a}}{\hbar \omega_{T}}+\frac{\beta_{L} b}{\exp \left[\frac{\hbar \omega_{L}}{(\tau-\tau) \Omega_{a}}\right]-1},
$$

when $\tau \geq \tau_{\mathrm{c}}$, where $u_{T 0}=\frac{\beta_{T} b}{\exp \left(\frac{\hbar \omega_{T}}{\tau_{\mathrm{c}} \Omega_{a}}\right)-1}$. The second term at the right hand side of $u_{L}$ is contributed by the first limiting speed $v_{T m}$. With eqs. (5) and (6), we are able to capture the displacement-stress curves and the velocity-stress curves for twinning partial dislocations from MD simulations, as given in Figure 2.

The two limiting speeds and the stress-velocity dependence captured by the phonon theory are not only seen in $\mathrm{Cu}$. We further investigate the velocity of twinning partials at $0 \mathrm{~K}$ for several other F.C.C. metals including $\mathrm{Al}, \mathrm{Au}, \mathrm{Ni}$, $\mathrm{Ag}, \mathrm{Pd}, \mathrm{Pt}$ and $\mathrm{Cu}$. Figure 6 shows the kinetics of twinning partial dislocations at the strain rate of $2 \times 10^{9} / \mathrm{s}$. Despite the significant difference in elastic properties and stacking fault energies among those materials, their twinning partial dislocations move in a similar way in response to applied shear stress. The travel distance as a function of linearly increasing stress shows two distinct stages (Figure 6(a)), resulted from the abrupt velocity jump at high stress level. The theoretical prediction from eq. (6) is also shown in Figure 6(a). In Figure 6(b), we present the stress-dependent velocity of twinning dislocations. As the theoretical prediction from eq. (5) and the results from MD simulations match so well, we only show the curve from theoretical prediction. It is convenient to identify the two speeding limits at different stress regimes from Figure 6(b). In addition to abstract the speed limit from the velocity-stress curves, we also calculate the two speed limits from the Mach cones. Figure 6(c) and (d) shows the Mach cones in the horizontal velocity field $v_{x}$ for $\mathrm{Cu}$ and $\mathrm{Ni}$, respectively. The correlation of the moving velocity with the shear wave and longitudinal wave speeds is demonstrated. In Table 2, we summarize our simulation results on the limiting velocity of twinning partial dislocations in several metals of different density and elastic properties, as well as the material parameters used to capture the stress-velocity curves from MD simulations. For convenience, we also show in Figure 6(e) the normalized subsonic and supersonic dislocation speeds for different F.C.C. metals. The denominators are the respective shear wave speed and longitudinal wave speed of the specific metal. At the strain rate of $2 \times 10 \%$ and $0 \mathrm{~K}$, the first limiting speed of twinning partial dislocations is slower than the shear wave speed in the F.C.C. metals investigated here. The second limiting speed is slightly greater than the speed of the longitudinal wave. It may be due to the fact that our simulations are done in a single crystal with twin boundaries. The bulk modulus of single crystals should be higher than their polycrystalline 

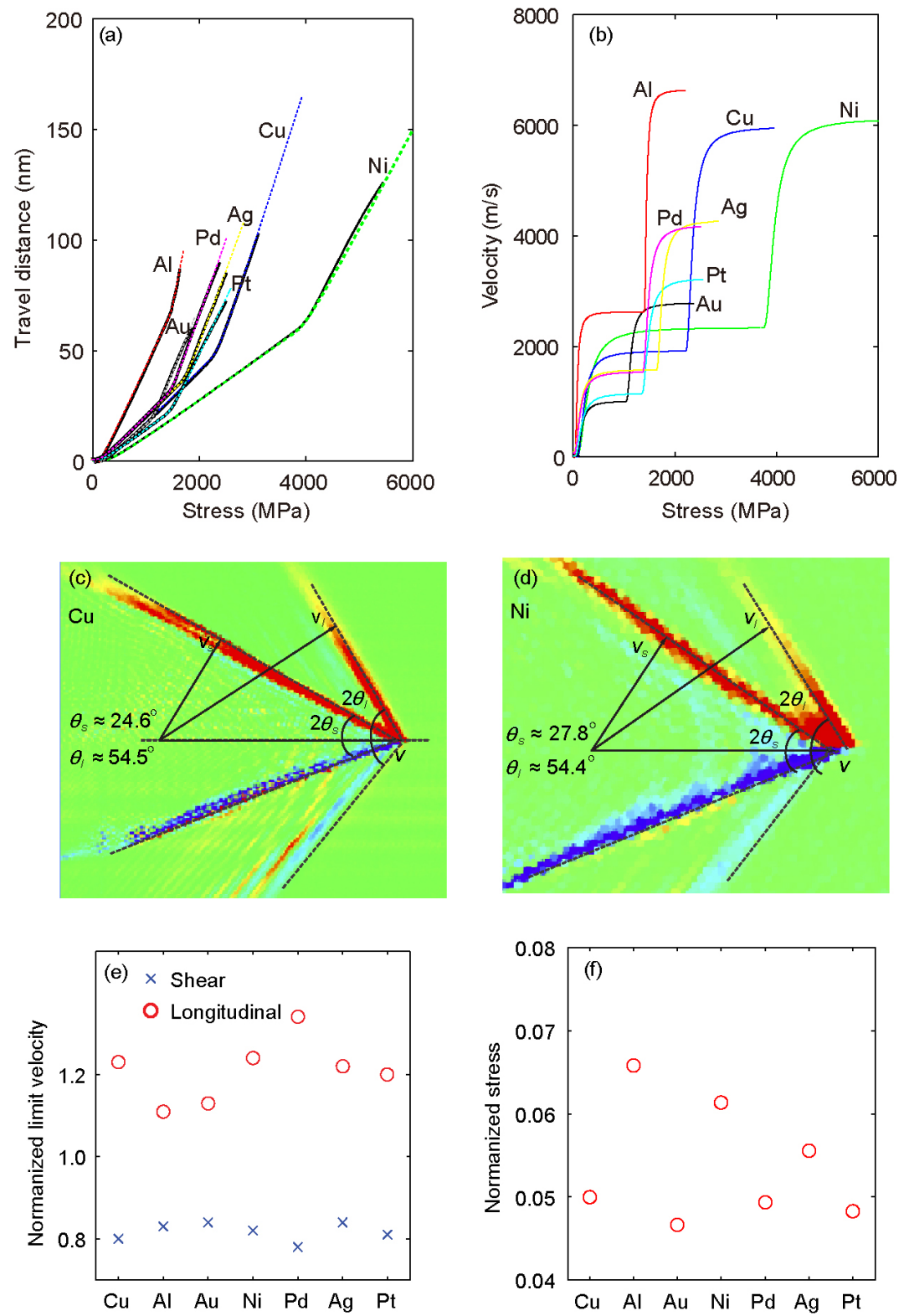

Figure 6 (Color online) The motion of twinning dislocations in different F.C.C. metals at the strain rate of $2 \times 10 \%$. (a) Travel distance as a function of linearly increased stress. Both results from MD simulations (solid line) and theoretical prediction (dotted line) are shown; (b) the velocity of twinning dislocations as a function of stress. As the data from MD simulations and theoretical predictions are highly repeatable, we only show the curves from theoretical prediction; (c) and (d) horizontal velocity field $v_{x}$ when dislocations moving at the second speed limit for $\mathrm{Cu}$ and $\mathrm{Ni}$, respectively, the longitudinal and shear wave speed can be calculated from the geometry of the Mach cone; (e) the normalized subsonic and supersonic speed of dislocations, and (f) the normalized transitional stress when velocity jump occurs.

counterparts, and the data we obtained from the literature are for latter case. In particular, disordered atoms in the grain boundaries of polycrystalline metals would enhance the compressibility of metals. Figure 6(f) shows the normalized shear stress (by the shear modulus of the metal) when the speed gap occurs. The typical critical stress for the transition is about $\tau_{\mathrm{c}} / G \approx 5 \%-7 \%$.

The solutions of linear elasticity theory predict no stable dislocation motion in the velocity regime $v_{\mathrm{s}}$ and $\sqrt{2} v_{\mathrm{s}}[14,19,44]$. Here we also observe the velocity gap from subsonic to supersonic motion of twinning partial dis- locations in F.C.C. metals, in accordance with previous MD calculations [7,16-18,33] and constitutive theories [53]. The ratio between the supersonic/subsonic speed limits, however, may be not exactly equal to $\sqrt{2}$. In our theory, the gap is resulted from the shift from transverse phonon assisted to longitudinal phonon assisted dislocation gliding. Hence the ratio of the supersonic limiting over the shear wave speed limit is determined by the ratio of the longitudinal wave speed $v_{1}$ and the shear wave speed $v_{\mathrm{s}}$ here. It is written as $\frac{v_{1}}{v_{\mathrm{s}}}=\sqrt{\frac{2(1-v)}{1-2 v}}$ for $v$ being the Poisson's ratio. We see that 
Table 2 Material properties of several typical F.C.C. metals at $0 \mathrm{~K}$ and their respective limiting velocities of a twinning partial dislocation (at the strain rate of $2 \times 10^{9} / \mathrm{s}$ ). Note that the two limiting speeds of each metal were calculated from the Mach cone of a dislocation moving except the longitudinal wave speeds of Al, Au, Pd, Pt. Their Mach cones are not sharp enough for precisely determination for the longitudinal wave speed. We used their theoretical calculations with $v_{1}{ }^{\mathrm{md}}=\sqrt{\left(B^{\mathrm{md}}+4 G^{\mathrm{md}} / 3\right) \rho}$, where $B^{\mathrm{md}}$ and $G^{\mathrm{md}}$ are obtained from MD simulations at zero-strain. Since $G^{\mathrm{md}}$ from the simulation varies slightly at large elastic strain, we supplied its range within the applied strain between 0 and $6 \%$. The two speed limits $v_{\mathrm{s}}, v_{1}$ come directly from MD simulations

\begin{tabular}{|c|c|c|c|c|c|c|c|c|c|c|c|c|c|c|c|c|}
\hline Parameter & $G^{\mathrm{md}}$ & $v$ & $\Omega_{a}$ & $b$ & $\rho$ & $v_{\mathrm{s}}$ & $v_{1}$ & $\begin{array}{c}\omega_{T} \text { or } \\
\omega_{T}\end{array}$ & $\beta_{T}$ & $\beta_{L}$ & $v_{\mathrm{s}}^{\mathrm{md}}$ & $v_{\mathrm{s}}^{\mathrm{md}} / v_{\mathrm{s}}$ & $v_{1}^{\mathrm{md}}$ & $v_{1}^{\mathrm{md}} / v_{1}$ & $\tau_{\mathrm{c}}$ & $\tau_{\mathrm{c}} / G$ \\
\hline Units & $\mathrm{GPa}$ & - & $\AA^{3}$ & $\AA$ & $\mathrm{kg} / \mathrm{m}^{3}$ & $\mathrm{~m} / \mathrm{s}$ & $\mathrm{m} / \mathrm{s}$ & $/ \mathrm{s}$ & - & - & $\mathrm{m} / \mathrm{s}$ & - & $\mathrm{m} / \mathrm{s}$ & - & $\mathrm{MPa}$ & - \\
\hline $\mathrm{Cu}$ & $36.5-41.0$ & 0.34 & 11.46 & 1.46 & 8933 & 2360 & 4610 & 0.35 & 13.3 & 26.5 & 1894 & 0.8 & 5667 & 1.23 & 2300 & 0.05 \\
\hline $\mathrm{Al}$ & $22.4-27.2$ & 0.35 & 16.56 & 1.65 & 2740 & 3170 & 6000 & 0.46 & 9.3 & 14.2 & 2636 & 0.83 & 6660 & 1.11 & 1500 & 0.068 \\
\hline $\mathrm{Au}$ & $19.5-23.0$ & 0.42 & 16.79 & 1.66 & 19265 & 1180 & 2460 & 1 & 8.5 & 15.3 & 992 & 0.84 & 2778 & 1.13 & 1050 & 0.05 \\
\hline $\mathrm{Pd}$ & 29.4-32.8 & 0.39 & 14.37 & 1.58 & 12010 & 1900 & 3700 & 0.42 & 6.9 & 12.6 & 1480 & 0.78 & 4202 & 1.34 & 1500 & 0.05 \\
\hline $\mathrm{Ag}$ & $27.1-31.6$ & 0.37 & 16.97 & 1.67 & 10474 & 1770 & 3390 & 0.6 & 7.7 & 15.3 & 1486 & 0.84 & 4146 & 1.22 & 1750 & 0.056 \\
\hline $\mathrm{Pt}$ & $30.0-32.4$ & 0.38 & 14.97 & 1.6 & 21517 & 1410 & 2690 & 0.65 & 7.3 & 12.8 & 1140 & 0.81 & 3240 & 1.2 & 1530 & 0.05 \\
\hline
\end{tabular}

even for isotropic materials, the ratio of supersonic speed over shear wave speed depends on the Poisson's ratio. For $v=0$, it approaches to the theoretical analysis of $v_{1} / v_{\mathrm{s}}=\sqrt{2}$. For $v=1 / 3, v_{1} / v_{\mathrm{s}}=2$.

\section{Conclusion and discussion}

In this paper, we employ large-scale molecular dynamics simulations to explore the velocity-stress relationship in a twinning partial dislocation in face centred cubic crystals. The choice for a twinning partial dislocation is two folds: (1) a complete dislocation in F.C.C metals in MD systems will split into two partials with stacking fault in between. We demonstrate in Figure 7(a) the atomic structure of a complete dislocation at equilibrium in $\mathrm{Cu}$. The corresponding stress field $\sigma_{x y}$ is shown in Figure 7(b). During their motion, the dissociated dislocations will have to drag a stacking fault along with them. Such a scenario would make it is difficult for theoretical analysis as we aim to obtain the velocity-stress relationship of a simple dislocation on which intensively theories have been developed. In contrast, a twinning partial dislocation is pure edge type, as seen in Figure 1. (2) Since the collective nucleation and propagation of twinning par- tial dislocations are generally considered to fulfil twin deformation, the limiting velocity of twinning partial dislocations could also be applied to understand the kinetics of deformation twinning in crystalline materials [54-56].

We demonstrate that a twinning partial dislocation exhibits two limiting speeds: the first is subsonic and occurs when the resolved shear stress is on the order of hundreds of megapascal. While the stress is raised to gigapascal level, an abrupt jump of dislocation velocity occurs. The first and second limiting speeds of the obstacle-free twinning partial dislocations are still subsonic in nature if compared to the transverse and longitudinal sound waves, respectively. If we adopt the definition proposed by Weertman [57], the second speed limit is supersonic. The speed of the twinning partial dislocation reach a saturated supersonic value which is about $20 \%$ above its longitudinal sound wave. Further increase in stress would lead to homogeneous nucleation of dislocations. The resolved shear stress to a particular dislocation is hence bounded as the single crystal yields. By adopting the Einstein model for the density of states and also the Planck distribution for the average number of phonons, we build the linkage of the two limiting speeds with phonon vibration assisted dislocation motion. The model not only captures the stress-dependent dislocation velocity, but also explains the physical
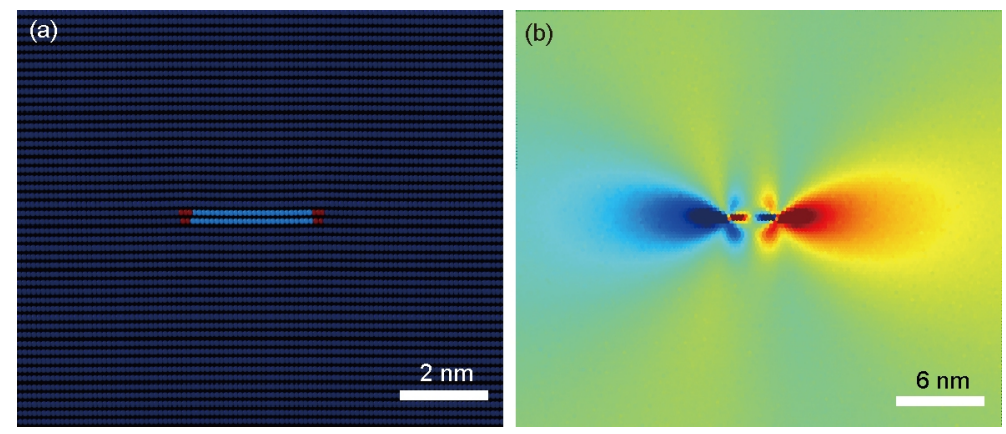

Figure 7 (Color online) The dissociation of a complete dislocation in F.C.C. Cu at equilibrium. (a) The atomic structure of the split complete dislocation; (b) the stress field introduced by the dissociated dislocations. 
origins of the two limiting speeds.

There could be remaining concerns about how well the empirical potentials may describe the interaction of atoms and the high strain rate employed here. Indeed, the embedded atom method (EAM) potentials used here are more powerful in capturing elastic-plastic coupled deformation like the motion of dislocation than class elastic theories: MD simulations with those potentials not only fully predict the anisotropic nature of crystals but also captures energy terms related to dislocation activities, like the stacking fault energies and the interfacial energies; existing isotropic elastic theories for dislocation motion are incapable of including those factors. For the concerns of high strain rates, we examined the motion of dislocations resulted from deformation rate. It is noted that at the strain rate of $\dot{\varepsilon}=2 \times 10 \%$, the artificial dislocation velocity imposed by the straining may reach $v=\dot{\varepsilon} h=2 \times 10 \% / \mathrm{s} \times 456 \mathrm{~nm} / 2=456 \mathrm{~m} / \mathrm{s}$. For $\dot{\varepsilon}=2 \times 10^{8} / \mathrm{s}$, it drops to $45.6 \mathrm{~m} / \mathrm{s}$, the speeds of dislocations are at least one order of magnitude faster. In particular, since we track the relative motion of oppositely moving dislocations in the sample seen in Figure 1(b), the straining induced velocity is automatically excluded. Our simulations for $\mathrm{Cu}$ at different strain rates demonstrate that the speed transition is robustly seen, regardless the slight variation in the exact number of the speeds. The difficulty in observing the second speed limit at the strain rate of $2 \times 10^{7} / \mathrm{s}$ is due to the limited sample size: before reaching the second speed limit, the two dislocations traveled a displacement greater than their initial distance, even we have used a cutting-on-edge simulation sample size of $1.8 \mathrm{~m}$ in width.

In summary, the dislocation kinetics model proposed here is the first kind to shed light on the microscopic mechanisms of the limiting factors for dislocation velocities. It could be directly applied to dislocation dynamics simulations [58-60] as physically faithful mobility laws. As the two speed limits occur at high stress level $\left(\tau_{\mathrm{c}} / G \approx 5 \%-7 \%\right)$, it may be commonly seen in fast deformation like metals under shock loads or during cracking. For example, the anomalous strain-rate sensitivity at high strain rates $\left(10^{3} / \mathrm{s}\right.$ and above) could be related to the transition in dislocation velocities during fast deformation $[61,62]$. We further note that the results reported here are for pure edge type twinning partial dislocations. It would be of great interest to see the dynamics of a screw partials or dislocation of mixing feature. As moving screw dislocation does not emit longitudinal waves, there exists no sound barrier between the subsonic motion and supersonic motion except for a single transonic state [44]. In addition, dislocations of mixed character are more complex because the contributions to core radiation from the edge component and the screw component are strongly coupled $[7,16,63]$. We leave this part in the future work.
China (Grant No. 11425211).

\section{Supporting Information}

The supporting information is available online at http://phys.scichina.com and http://link.springer.com/journal/11433. The supporting materials are published as submitted, without typesetting or editing. The responsibility for scientific accuracy and content remains entirely with the authors.

1 G. I. Taylor, Proc. R. Soc. A 145, 362 (1934).

2 M. Polanyi, Z. Physik 89, 660 (1934).

3 E. Orowan, Z. Physik 89, 605 (1934).

4 M. A. Meyer, Dynamic Behavior of Materials (John Wiley \& Sons Inc., Hoboken, 1994), p. 323.

5 A. S. Argon, Strengthening Mechanisms in Crystal Plasticity (Oxford University Press, Oxford, 2007).

6 A. J. Rosakis, O. Samudrala, and D. Coker, Science 284, 1337 (1999).

7 P. Gumbsch, and H. Gao, Science 283, 965 (1999).

8 J. R. Rice, J. Mech. Phys. Solids 40, 239 (1992).

9 C. C. Chen, C. Zhu, E. R. White, C. Y. Chiu, M. C. Scott, B. C. Regan, L. D. Marks, Y. Huang, and J. Miao, Nature 496, 74 (2013).

10 J. N. Clark, J. Ihli, A. S. Schenk, Y. Y. Kim, A. N. Kulak, J. M. Campbell, G. Nisbet, F. C. Meldrum, and I. K. Robinson, Nat. Mater. 14, 780 (2015), arXiv: 1501.02853.

11 W. G. Johnston, and J. J. Gilman, J. Appl. Phys. 30, 129 (1959).

12 S. Schäfer, Phys. Stat. Solidi 19, 297 (1967).

13 F. C. Frank, Proc. Phys. Soc. A 62, 131 (1949).

14 J. D. Eshelby, Proc. Phys. Soc. A 62, 307 (1949).

15 Y. Y. Earmme, and J. H. Weiner, J. Appl. Phys. 45, 603 (1974).

16 D. L. Olmsted, L. G. Hectorjr, W. A. Curtin, and R. J. Clifton, Model. Simul. Mater. Sci. Eng. 13, 371 (2005).

17 Z. Jin, H. Gao, and P. Gumbsch, Phys. Rev. B 77, 094303 (2008).

18 H. Tsuzuki, P. S. Branicio, and J. P. Rino, Acta Mater. 57, 1843 (2009).

19 P. Rosakis, Phys. Rev. Lett. 86, 95 (2001).

20 V. Nosenko, S. Zhdanov, and G. Morfill, Phys. Rev. Lett. 99, 025002 (2007), arXiv: 0709.1782.

21 E. Faran, and D. Shilo, Phys. Rev. Lett. 104, 155501 (2010).

22 V. Nosenko, G. E. Morfill, and P. Rosakis, Phys. Rev. Lett. 106, 155002 (2011), arXiv: 1105.0614

23 J. J. Gilman, Metall. Mat. Trans. A 31, 811 (2000).

24 W. F. Greenman, T. Vreeland Jr., and D. S. Wood, J. Appl. Phys. 38, 3595 (1967).

25 K. Yasutake, S. Shimizu, M. Umeno, and H. Kawabe, J. Appl. Phys. 61, 940 (1987).

26 F. F. Abraham, R. Walkup, H. Gao, M. Duchaineau, T. Diaz de La Rubia, and M. Seager, Proc. Natl. Acad. Sci. USA 99, 5783 (2002).

27 F. F. Abraham, R. Walkup, H. Gao, M. Duchaineau, T. Diaz de La Rubia, and M. Seager, Proc. Natl. Acad. Sci. USA 99, 5777 (2002).

28 S. Yip, Nat. Mater. 3, 11 (2004).

29 M. J. Buehler, and H. J. Gao, Nature 439, 307 (2006).

30 T. Zhu, J. Li, A. Samanta, A. Leach, and K. Gall, Phys. Rev. Lett. 100, 025502 (2008).

31 X. Li, Y. Wei, L. Lu, K. Lu, and H. Gao, Nature 464, 877 (2010).

32 J. P. Hirth, and J. Lothe, Theory of Dislocations (Krieger Publishing Company, Malabar, 1982).

33 N. Bhate, R. J. Clifton, and R. Phillips, AIP Conf. Proc. 620, 339 (2002).

34 S. Plimpton, J. Comp. Phys. 117, 1 (1995).

35 M. S. Daw, and M. I. Baskes, Phys. Rev. B 29, 6443 (1984).

36 Y. Mishin, M. J. Mehl, D. A. Papaconstantopoulos, A. F. Voter, and 
J. D. Kress, Phys. Rev. B 63, 224106 (2001).

37 X. W. Zhou, H. N. G. Wadley, R. A. Johnson, D. J. Larson, N. Tabat, A. Cerezo, A. K. Petford-Long, G. D. W. Smith, P. H. Clifton, R. L. Martens, and T. F. Kelly, Acta Mater. 49, 4005 (2001).

38 S. M. Foiles, M. I. Baskes, and M. S. Daw, Phys. Rev. B 33, 7983 (1986).

39 R. Peierls, Proc. Phys. Soc. 52, 34 (1940).

40 F. R. N. Nabarro, Mater. Sci. Eng.-A 234-236, 67 (1997).

41 J. A. Gorman, D. S. Wood, and T. Vreeland Jr., J. Appl. Phys. 40, 833 (1969).

42 J. J. Gilman, Micromechanics of Flow in Solids (McGraw-Hill, New York, 1969).

43 T. Ninomiya, J. Phys. Soc. Jpn. 25, 830 (1968).

44 J. Weertman, and J. R. Weertman, Moving Dislocations, edited by F. R. N. Nabarro (North-Holland Publishing Company, Oxford, 1980), pp. $1-59$.

45 M. Planck, Ann. Phys.-Berlin 4, 553 (1901).

46 A. Einstein, Ann. Phys.-Berlin 22, 569 (1907).

47 V. Celli, and N. Flytzanis, J. Appl. Phys. 41, 4443 (1970).

48 S. Ishioka, J. Phys. Soc. Jpn. 30, 323 (1971).

49 O. Kresse, and L. Truskinovsky, J. Mech. Phys. Solids 51, 1305 (2003).

50 O. Kresse, and L. Truskinovsky, J. Mech. Phys. Solids 52, 2521 (2004).

51 G. I. Taylor, and H. Quinney, Proc. R. Soc. A 143, 307 (1934).

52 J. J. Mason, A. J. Rosakis, and G. Ravichandran, Mech. Mater. 17, 135 (1994).

53 X. Zhang, A. Acharya, N. J. Walkington, and J. Bielak, J. Mech. Phys. Solids 84, 145 (2015).

54 L. Lu, X. Chen, X. Huang, and K. Lu, Science 323, 607 (2009).

55 O. Grässel, L. Krüger, G. Frommeyer, and L. W. Meyer, Int. J. Plasticity 16, 1391 (2000).

56 Y. Li, L. Zhu, Y. Liu, Y. Wei, Y. Wu, D. Tang, and Z. Mi, J. Mech. Phys. Solids 61, 2588 (2013).

57 J. Weertman, J. Appl. Phys. 38, 5293 (1967).

58 W. Cai, and V. V. Bulatov, Mater. Sci. Eng.-A 387-389, 277 (2004).

59 B. Devincre, T. Hoc, and L. Kubin, Science 320, 1745 (2008).

60 K. Kang, V. V. Bulatov, and W. Cai, Proc. Natl. Acad. Sci. USA 109, 15174 (2012).

61 U. S. Lindholm, Deformation Maps in the Region of High Dislocation Velocity (Springer Berlin Heidelberg, Berlin, 1979).

62 K. Kadau, T. C. Germann, P. S. Lomdahl, and B. L. Holian, Science 296, 1681 (2002).

63 Z. H. Jin, P. Gumbsch, E. Ma, K. Albe, K. Lu, H. Hahn, and H. Gleiter, Scripta. Mater. 54, 1163 (2006).

64 C. Kittel, Introduction to Solid State Physics, 8th ed (Wiley, Hoboken, 2005).

\section{Appendix}

Here, we build the connection between stress and temperature on how they may alter phonon distribution. It has been well known that the anharmonic terms accounts for the thermal properties of materials [64]. For simplicity but without loss of generality, we consider a pair potential with a minimum energy of $u_{0}$. Its harmonic approximation, which represents the materials at very small elastic deformation quite well, will deviate from the real energy profile perceivably. Such asymmetrical potential gives rise to transportation of phonons, and results in the flux of heat. Such a concept is well known and is documented in commonly seen textbooks. By expanding the potential function near the equilibrium position $\left(\delta=r-r_{m}\right)$, and include higher order terms than the harmonic approximation owes, we have

$u(r)=u_{0}+K \frac{\delta^{2}}{2}+\psi \frac{\delta^{3}}{6}+\chi \frac{\delta^{4}}{24}+O\left(\delta^{4}\right)$.

In the above equation, we have defined $K=\frac{\mathrm{d}^{2} u(r)}{\mathrm{d}^{2} r}$, $\psi=\frac{\mathrm{d}^{3} u(r)}{\mathrm{d}^{3} r}$, and $\chi=\frac{\mathrm{d}^{4} u(r)}{\mathrm{d}^{4} r}$ at $r=r_{m}$, and used the factor $\frac{\mathrm{d} u(r)}{\mathrm{d} r}=0$ at the equilibrium position. Terms from the third one on the right hand side of eq. (a1) are related to the anharmonic nature of the interaction, from which thermal expansion originates. Specifically, the coefficient $\psi$ weights the asymmetry of the mutual repulsion of the atoms and $\chi$ represents the softening of the vibration at large amplitudes [64]. Following the Boltzmann distribution, the linear expansion of lattice at thermal equilibrium will lead to an elongation of lattice size by an amount of $\bar{\delta}$, which is determined as:

$\bar{\delta}=\frac{\int_{-r_{m}}^{\infty} \delta \mathrm{e}^{-\frac{u\left(r_{m}+\delta\right)}{k_{\mathrm{B}} T}} \mathrm{~d} \delta}{\int_{-r_{m}}^{\infty} \mathrm{e}^{-\frac{u\left(r_{m}+\delta\right)}{k_{\mathrm{B}} T}} \mathrm{~d} \delta} \approx-\frac{1}{2} \frac{\psi}{K^{2}} k_{\mathrm{B}} T$,

where $k_{\mathrm{B}}$ is the Boltzmann constant; $T$ is the absolute temperature.

At the same time, when we exert a force to pull the atomic bond apart, the lattice will expand as well. The force-displacement relationship is given by $f(r)=\frac{\mathrm{d} u(r)}{\mathrm{d} r}$. Using the expanded expression for $u(r)$, we have

$f(\delta)=K \delta+\psi \frac{\delta^{2}}{2}+\chi \frac{\delta^{3}}{6}$.

Let $S_{a}$ the planar footprint of the atom with surface normal parallel to the loading direction. We may obtain the stress $\sigma$ as a function of lattice extension $\delta$ :

$\sigma=\frac{f(\delta)}{S_{a}}=\frac{1}{S_{a}}\left(K \delta+\psi \frac{\delta^{2}}{2}+\chi \frac{\delta^{3}}{6}\right)$.

Now $\delta$ can be expressed in terms of the applied stress $\sigma$. If we take the linear term for $\sigma$ vs. $\delta$, then

$\delta=\frac{\sigma S_{a}}{K}$.

Inputting eq. (a5) into eq. (a2) and recognizing the equivalence of $\delta$ and $\bar{\delta}$, we have

$\frac{\sigma S_{a}}{K}=-\frac{1}{2} \frac{\psi}{K^{2}} k_{\mathrm{B}} T$.

From which we obtain the following relationship between $\sigma$ and $T$ :

$\sigma=-\frac{1}{2} \frac{\psi}{S_{a} K} k_{\mathrm{B}} T$ 
Note that lattice "expansion" by uniaxial stress occurs along one direction but thermal expansion is typically three-dimensional. As the motion of dislocations is mostly sensitive to the distance of the gliding planes in comparison with the other two directions, the relationship proposed in eq. (a6) seems to be reasonable.

Now we consider the commonly seen pair potential in physical community to express eq. (a6) in parameters we are familiar with. Let's consider a Lennard-Jones interaction

$u(r)=4 u_{0}\left[\left(\frac{r_{0}}{r}\right)^{12}-\left(\frac{r_{0}}{r}\right)^{6}\right]$ where $u_{0}$ is the cohesive energy. Its Taylor expansion at the equilibrium point $r_{m}=\sqrt[6]{2} r_{0}$ in the form of eq. (a1) leads to

$$
\begin{aligned}
u\left(r_{m}+\delta\right)= & -u_{0}+36 u_{0}\left(\frac{\delta}{r_{m}}\right)^{2}-252 u_{0}\left(\frac{\delta}{r_{m}}\right)^{3} \\
& +1113 u_{0}\left(\frac{\delta}{r_{m}}\right)^{4}+O\left(\delta^{4}\right) .
\end{aligned}
$$

Hence we have $K=72 u_{0} / r_{m}^{2}, \quad \psi=-1512 u_{0} / r_{m}^{3}$, and $\chi=26712 u_{0} / r_{m}^{4}$. With eq. (a6), we have $\sigma=\frac{21 k_{\mathrm{B}} T}{S_{a} r_{m}}$, where $S_{d} r_{m}$ is approximate to the atomic volume. 